\title{
Whole genome sequence and de novo assembly revealed genomic architecture of Indian Mithun (Bos frontalis)
}

\author{
Sabyasachi Mukherjee ${ }^{1 * \dagger} \mathbb{D}$, Zexi Cai ${ }^{2 \dagger}$, Anupama Mukherjee ${ }^{1,3}$, Imsusosang Longkumer ${ }^{1}$, Moonmoon Mech', \\ Kezhavituo Vupru' ${ }^{1}$ Kobu Khate ${ }^{1}$, Chandan Rajkhowa' ${ }^{1}$, Abhijit Mitra', Bernt Guldbrandtsen², \\ Mogens Sandø Lund ${ }^{2}$ and Goutam Sahana ${ }^{2}$
}

\begin{abstract}
Background: Mithun (Bos frontalis), also called gayal, is an endangered bovine species, under the tribe bovini with $2 n=58 X X$ chromosome complements and reared under the tropical rain forests region of India, China, Myanmar, Bhutan and Bangladesh. However, the origin of this species is still disputed and information on its genomic architecture is scanty so far. We trust that availability of its whole genome sequence data and assembly will greatly solve this problem and help to generate many information including phylogenetic status of mithun. Recently, the first genome assembly of gayal, mithun of Chinese origin, was published. However, an improved reference genome assembly would still benefit in understanding genetic variation in mithun populations reared under diverse geographical locations and for building a superior consensus assembly. We, therefore, performed deep sequencing of the genome of an adult female mithun from India, assembled and annotated its genome and performed extensive bioinformatic analyses to produce a superior de novo genome assembly of mithun.
\end{abstract}

Results: We generated $\approx 300$ Gigabyte $(\mathrm{Gb})$ raw reads from whole-genome deep sequencing platforms and assembled the sequence data using a hybrid assembly strategy to create a high quality de novo assembly of mithun with $96 \%$ recovered as per BUSCO analysis. The final genome assembly has a total length of $3.0 \mathrm{~Gb}$, contains 5,015 scaffolds with an N50 value of $1 \mathrm{Mb}$. Repeat sequences constitute around $43.66 \%$ of the assembly. The genomic alignments between mithun to cattle showed that their genomes, as expected, are highly conserved. Gene annotation identified 28,044 protein-coding genes presented in mithun genome. The gene orthologous groups of mithun showed a high degree of similarity in comparison with other species, while fewer mithun specific coding sequences were found compared to those in cattle.

Conclusion: Here we presented the first de novo draft genome assembly of Indian mithun having better coverage, less fragmented, better annotated, and constitutes a reasonably complete assembly compared to the previously published gayal genome. This comprehensive assembly unravelled the genomic architecture of mithun to a great extent and will provide a reference genome assembly to research community to elucidate the evolutionary history of mithun across its distinct geographical locations.

Keywords: Mithun, Bos frontalis, Genome, de novo assembly

\footnotetext{
* Correspondence: smup0336@gmail.com

+Sabyasachi Mukherjee and Zexi Cai contributed equally to this work.

${ }^{1}$ Animal Genetics and Breeding Lab., ICAR-National Research Centre on

Mithun, Medziphema, Nagaland 797106, India

Full list of author information is available at the end of the article
}

(c) The Author(s). 2019 Open Access This article is distributed under the terms of the Creative Commons Attribution 4.0 International License (http://creativecommons.org/licenses/by/4.0/), which permits unrestricted use, distribution, and reproduction in any medium, provided you give appropriate credit to the original author(s) and the source, provide a link to the Creative Commons license, and indicate if changes were made. The Creative Commons Public Domain Dedication waiver (http://creativecommons.org/publicdomain/zero/1.0/) applies to the data made available in this article, unless otherwise stated. 


\section{Background}

Mithun (Bos frontalis) is a rare bovine species living under free-range conditions inside tropical rainforest ecosystems of India, Bangladesh, Bhutan, China, and Myanmar [1]. It is a unique animal having a massive body, with characteristic 'white stockings' on their stout legs. This animal efficiently converts grass, forage, tree leaves as well as various agricultural by-products into highly nutritious meat. Moreover, mithun holds a unique place in the evolution of bovines. Mithun, having a specific chromosomal pattern, $2 \mathrm{n}=58$ is distinguishable from that of cattle $(2 n=60)$ and yak $(2 n=60)$ [2]. However, the origin of mithun is an on-going debate with no well-supported conclusion [3-5]. The deviation of the karyotype maybe originated from a $2 / 27$ centric fusion or a Robertsonian translocation of cattle chromosomes 2 and 28 [6]. Besides ambiguity on its origin, information on genomic architecture of mithun is scanty so far.

Recently, researchers have carried out genomic studies on mithun. Mai et al. [7] reported whole-genome sequencing of mithun to detect single nucleotide polymorphisms (SNPs), copy number variations (CNVs), structural variations (SVs), SNP annotation and functional enrichment analysis of non-synonymous SNPs. Another research group presented the first genome assembly for gayal (mithun of Chinese origin) [8]. However, it is valuable to obtain genome sequence of individual from another geographical location to have better understanding on the genomic variation of mithun, which may also help to build a consensus assembly.

With rapid progress in sequencing technologies like next-generation sequencing (NGS) platforms, whole genomes of most livestock species have been sequenced to discover the underlying genetic architecture and explore species diversity, construct haplotype maps and perform genome-wide association studies. De novo assembly of many bovinae genomes including taurine cattle [9], indicine cattle [10], water buffalo [11], yak [12] and gayal [8] have been completed. Genome comparisons between closely related species provide insights into the genetic basis of mammalian divergence and adaptation [12]. Here we performed whole genome sequencing of one Indian adult female mithun $(2 \mathrm{n}=58, \mathrm{XX})$ using multiple sequencing platforms (Illumina HiSeq, Illumina Moleculo long reads and Pacific Biosciences: PacBio) [13-15] to generate a de novo genome assembly. This assembly was compared with genome assemblies of other species in the tribe bovini including the published gayal genome assembly [8]. We believe that an improved reference genome assembly would benefit understanding genetic divergence in mithun populations reared under diverse geographical locations and would be helpful in understanding the genomic architecture of this species.

\section{Results}

\section{Genome assembly and assessment}

Using multiple sequencing platforms, we generated a total of $241 \mathrm{~Gb}$ Illumina pair-end reads and mate-pair reads, 4.4 Gb Moleculo long reads and 4.8 Gb PacBio reads after removing adapter sequences and low quality reads. We adopted a hybrid strategy to assemble the genome. First, all the contaminating adapter sequences from the Illumina reads were removed, and then, the reads with low quality bases were trimmed. After that, paired-end reads were pre-assembled by an open access hybrid assembler MaSuRCA [16]. Next, these pre-assembled contigs were arranged in order and assembled into scaffolds by open-access script in SSPACE [17], combining with mate-pairs information. Finally, scaffolding was repeated by combining with Moleculo long reads and PacBio reads (corrected by LoRDEC [18]) by SSPACE-Long Read [19]. Genome size of mithun was estimated to be $3.09 \mathrm{~Gb}$ and $3.00 \mathrm{~Gb}$ was recovered in our assembly. A previous report estimated the genome size of gayal as $3.15 \mathrm{~Gb}$ and assembled the $2.85 \mathrm{~Gb}$ sequence with $\mathrm{N} 50$ value of $2.74 \mathrm{Mb}$ [8]. Our genome assembly consists of 5,015 scaffolds. The size of the largest scaffold was $6,540,552 \mathrm{bp}$ with N50 value as 1.00 $\mathrm{Mb}$. The assembly of mithun genome presented here is less fragmented (5,015 vs. 460,059 scaffolds) and is more complete than the previously published gayal genome assembly [8] (Table 1, Fig. 1). Figure 1 showed that our mithun genome assembly was evenly distributed across the length of scaffolds. To assess the correctness of our assembly, we aligned paired-end reads and mate-pair reads onto the assembled mithun genome. The result showed $98.70 \%$ of the mate-pair reads could be aligned to our mithun genome assembly, $82.99 \%$ for $3 \mathrm{~kb}$ library and $84.42 \%$ of $5 \mathrm{~kb}$ library are properly paired with the mithun assembly (Table 2 ). This result proved a high degree of correct ordering and orientation of sequences in our mithun genome assembly. To check whether the genome included most of the protein coding genes, a

Table 1 Summary details of the mithun genome assembly compared with gayal genome

\begin{tabular}{lll}
\hline & Mithun genome & Gayal genome [8] \\
\hline Total assembly length & $3.00 \mathrm{~Gb}$ & $2.85 \mathrm{~Gb}$ \\
Data Volume (after QC) & $250 \mathrm{~Gb}$ & $276 \mathrm{~Gb}$ \\
Contig count & 480,463 & 583,373 \\
Contig N50 & $11.5 \mathrm{~Kb}$ & $14.4 \mathrm{~Kb}$ \\
Scaffold count & 5,015 & 460,059 \\
Largest scaffold & $6.54 \mathrm{Mb}$ & $13 \mathrm{Mb}$ \\
Scaffold N50 & $1.00 \mathrm{Mb}$ & $2.74 \mathrm{Mb}$ \\
Gap count & 301,766 & $2,647,378$ \\
Total gap length & $106 \mathrm{Mb}$ & $421 \mathrm{Mb}$ \\
\hline
\end{tabular}




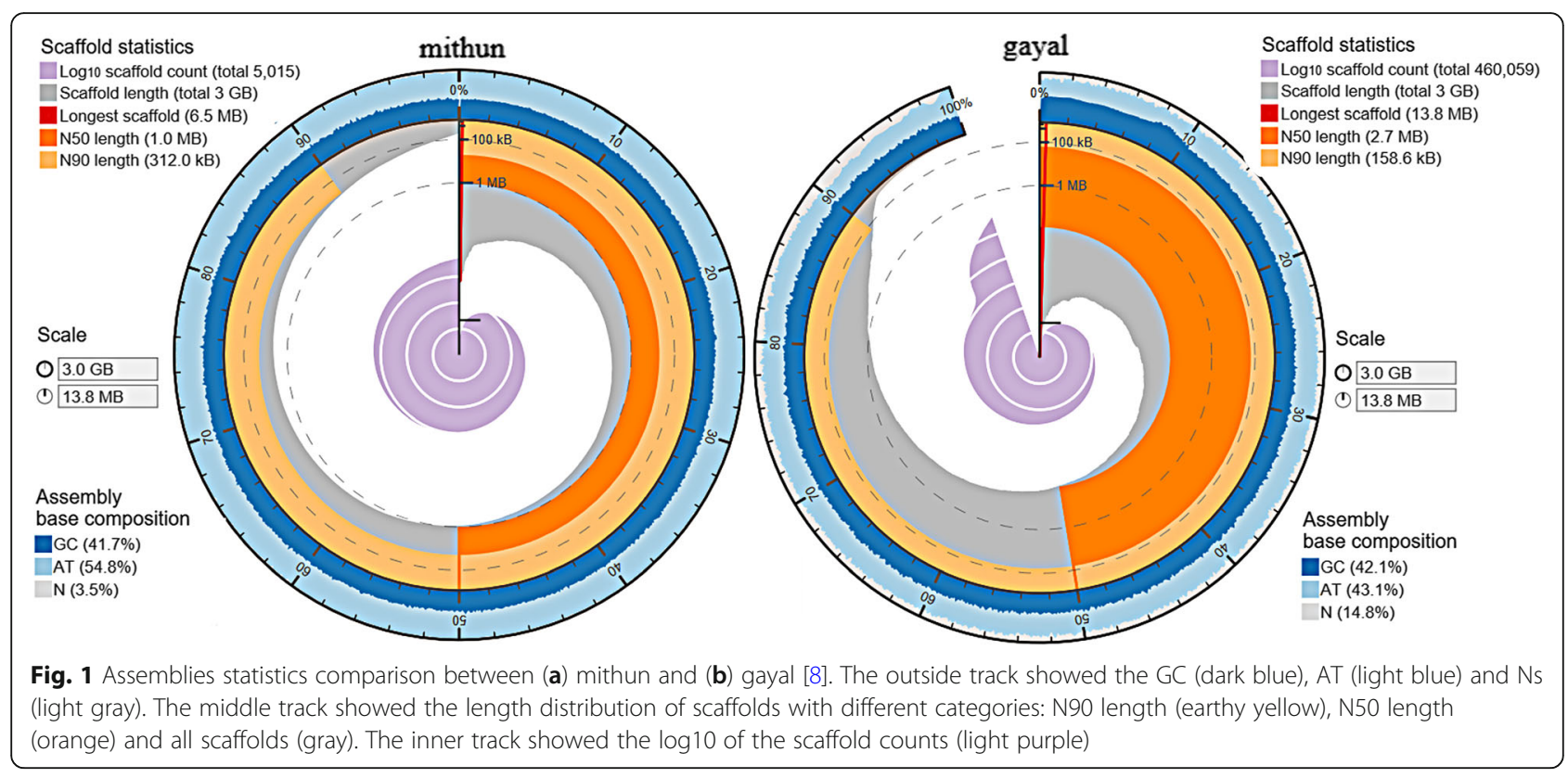

BUSCO [20] analysis was performed. It was found that $91.50 \%$ of genes found in other Bos species were completely covered, while only $4.1 \%$ of genes were not present in the mithun assembly (Table 3). Compared with previous gayal assembly [8], our mithun assembly recovered more genes, which indicated our assembly has better coverage.

\section{Repeat annotation}

We applied the RepeatMasker program [23] using the mammalian repeat database [24] to screen DNA sequences for repeats. The result showed the mithun genome harbors $43.66 \%$ of repeat sequences, comparable to $49.38 \%$ in cattle genome (pre-analysis genome from http://www.repeatmasker.org/) and the previous gayal assembly (48.13\%) [8]. The most abundant family was Long Interspersed Nuclear Elements (LINEs), followed by Short Interspersed Nuclear Elements (SINEs), which are common in mammalian genomes. Details of the genome proportion in each families are presented in Table 4. The substitution level of repeat sequences was estimated in mithun and compared with the cattle genome. As shown in Fig. 2, two genomes have similar pattern in the old repeat copies (number of substitutions greater than

Table 2 Sequence alignment of pair-end (PE) library, $3 \mathrm{~Kb}$ mate-pair (MP) library and $5 \mathrm{~Kb}$ MP library to assembly

\begin{tabular}{llll}
\hline & $\mathrm{PE}$ & $3 \mathrm{~Kb}$ & $5 \mathrm{~Kb}$ \\
\hline Reads aligned & $96.24 \%$ & $98.71 \%$ & $98.70 \%$ \\
Singletons & $0.26 \%$ & $0.87 \%$ & $0.92 \%$ \\
Properly paired & $90.81 \%$ & $82.99 \%$ & $84.42 \%$ \\
Mapped to different scaffolds & $0.05 \%$ & $13.86 \%$ & $14.34 \%$ \\
\hline
\end{tabular}

20; more the substitutions, greater the age of the copy of the element). In contrast, differences were observed in more recently inserted elements with fewer substitutions compared to the consensus. Mithun genome has a high peak between 5 and 15 substitutions and a smaller peak with between 0 and 5 . In contrast, most cattle repeat elements had between 0 and 5 , and a small peak between 5 and 15 (Fig. 2). These differences originate from LINEs and SINEs in the genome.

\section{Gene annotation}

Homology search, ab initio gene finding and transcriptome assembly were used to identify protein-coding genes. We identified 28,044 protein-coding genes in the mithun genome. Protein coding genes of orthologous groups were assigned by evolutionary genealogy of genes utilizing Non-supervised Orthologous Groups (eggNOG) mapper service [25], a public resource. We assigned 24, 755 mithun genes to 15,491 orthologous groups. The orthologous groups of mithun were compared with those in human, mouse, dog and cattle genome (Fig. 3). As expected, several orthologous groups were only shared between cattle and mithun (241 in total). This number was much higher than those with other species (mithun vs. human: 146, mithun vs. mouse: 28 , mithun vs. dog: 60). The domain of each protein coding sequence was scanned by InterProScan, an integration platform for the signature-recognition methods in InterPro [26], and 26,884 of 28,044 protein coding sequences were found to have at least one domain hit (Additional file 2: Table S1). The eggNOG mapper service [25] assigned possible gene names and the Gene Ontology (GO) [27] entries. A total of 26,041 sequences 
Table 3 BUSCO gene completeness assessment

\begin{tabular}{llllll}
\hline & Mithun & Bos taurus [21] & Bos indicus [22] & Bos grunniens [12] & Gayal [8] \\
\hline Complete & $91.5 \%$ & $92.3 \%$ & $90.1 \%$ & $93.6 \%$ & $85.2 \%$ \\
Fragments & $4.4 \%$ & $3.8 \%$ & $3.8 \%$ & $3.4 \%$ & $7.8 \%$ \\
Missing & $4.1 \%$ & $3.9 \%$ & $6.1 \%$ & $3.0 \%$ & $7.0 \%$ \\
\hline
\end{tabular}

had hits in the eggNOG database. Among these, 22,107 had GO entries (Additional file 3: Table S2). We performed a Kyoto Encyclopedia of Genes and Genomes (KEGG) pathway [28] analysis by KEGG Automatic Annotation Server (KAAS) [29]. KEGG entries could be assigned to 11,725 genes (Additional file 4: Table S3).

\section{Genome alignment}

Chromosome rearrangements between the mithun/gayal and cattle genomes were detected by aligning the mithun assembly to the cattle genome. $2.60 \mathrm{~Gb}$ of the $2.66 \mathrm{~Gb}$ cattle genome (leaving out Mt., Y chromosomes and unassigned sequences) aligned with the mithun sequence (98\% alignment). We also aligned previously published gayal assembly [8] to cattle genome, and 2.62 $\mathrm{Gb}$ of the $2.66 \mathrm{~Gb}$ cattle genome could be covered (98\%). As reflected by the N50 (Table 1) and scaffold size distribution (Fig. 1), the majority $(6,288$ of 15,089$)$ of alignment blocks of our mithun assembly were having most synteny blocks ranging between $100 \mathrm{~Kb} \sim 1 \mathrm{Mb}$. In total, they covered $1.87 \mathrm{~Gb}$ of sequence (Table 5). In gayal assembly [8], there are more synteny blocks belong to $1 \mathrm{Mb}$ to $10 \mathrm{Mb}$ block size. However, the gayal assembly have more small synteny blocks compared with mithun assembly. As shown in Fig. 4a, the four longest scaffolds aligned with many cattle chromosomes (alignments longer than $100 \mathrm{~Kb}$ ) and some of these scaffolds aligned to two chromosomes. The four longest scaffolds of gayal also had the similar alignment pattern as mithun (Fig. 4b). We also checked the genome alignment with cattle chromosomes 2, 27 and 28 to find any relic of chromosome fusion (Fig. $4 \mathrm{c}$ and d). If one of the

Table 4 The repeat sequence composition in the mithun genome

\begin{tabular}{lll}
\hline Family & Percent of genome & Copy number of elements \\
\hline LINEs & 23.48 & $1,559,559$ \\
SINEs & 11.84 & $2,413,359$ \\
LTR elements & 4.69 & 438,811 \\
Transposon & 2.17 & 313,666 \\
Small RNA & 1.60 & 282,842 \\
Satellites & 1.29 & 89,091 \\
Simple repeats & 0.73 & 549,262 \\
Low complexity & 0.14 & 88,687 \\
Unclassified & 0.02 & 3,858 \\
Total & 43.66 & \\
\hline
\end{tabular}

mithun chromosome is fusion of cattle chromosome 2 and 27 or chromosome 2 and 28, we should see a scaffold span the fusion site from two cattle chromosomes. However, we did not find clear evidence to support this hypothesis due to the fragmentation of both assembly.

\section{Discussion}

\section{Motivation for mithun genome assembly}

Mithun lives under free-range conditions in the tropical rain forests of the North Eastern Hill region of India, at an altitude ranging from 300 to $3,000 \mathrm{~m}$ above mean sea level. Mithun have great socio-economic importance among the mostly tribal population of the region. Mithun is primarily reared for meat, without any human inputs except occasional salt offerings [30]. Meat and milk of mithun have high quality in terms of higher fat $\%$ in milk and better marbling of its meat compared to cattle [31]. Besides, mithun may be resistant to mad cow disease [32]. These traits make mithun interesting for livestock research and for breeding. Even if the genome assembly of one gayal (mithun of Chinese origin) was recently published [8], the Indian mithun we sequenced here is from a diverse geographical location and separated for long time from the gayal animal sequenced previously [8]. Hence, we expected a high genome divergence between these two animals of different geographical origin. A superior genome assembly of mithun (Indian origin) will provide valuable information for research into mithun biology and genetics.

\section{Genome of Indian mithun}

Here we presented the first de novo genome assembly of Indian mithun, which is more complete, less fragmented and better annotated (96\%). By combining several next generation sequencing technologies, including some which generate long reads, we can avoid biases inherent in the individual technologies [33]. We used a hybrid genome assembly approach combining second and third generation sequencing data (combining short pair-end Illumina reads and Moleculo \& PacBio long sequence reads) to produce an assembly with better genome coverage, fewer gaps and better scaffold statistics. It was also reported that performance of genome assembly improves significantly from hybrid approach using both short and long sequence reads [34]. Simultaneously, this approach also keeps overall costs of the projects manageable [35-39]. Some genomes viz. human [37], Korean 


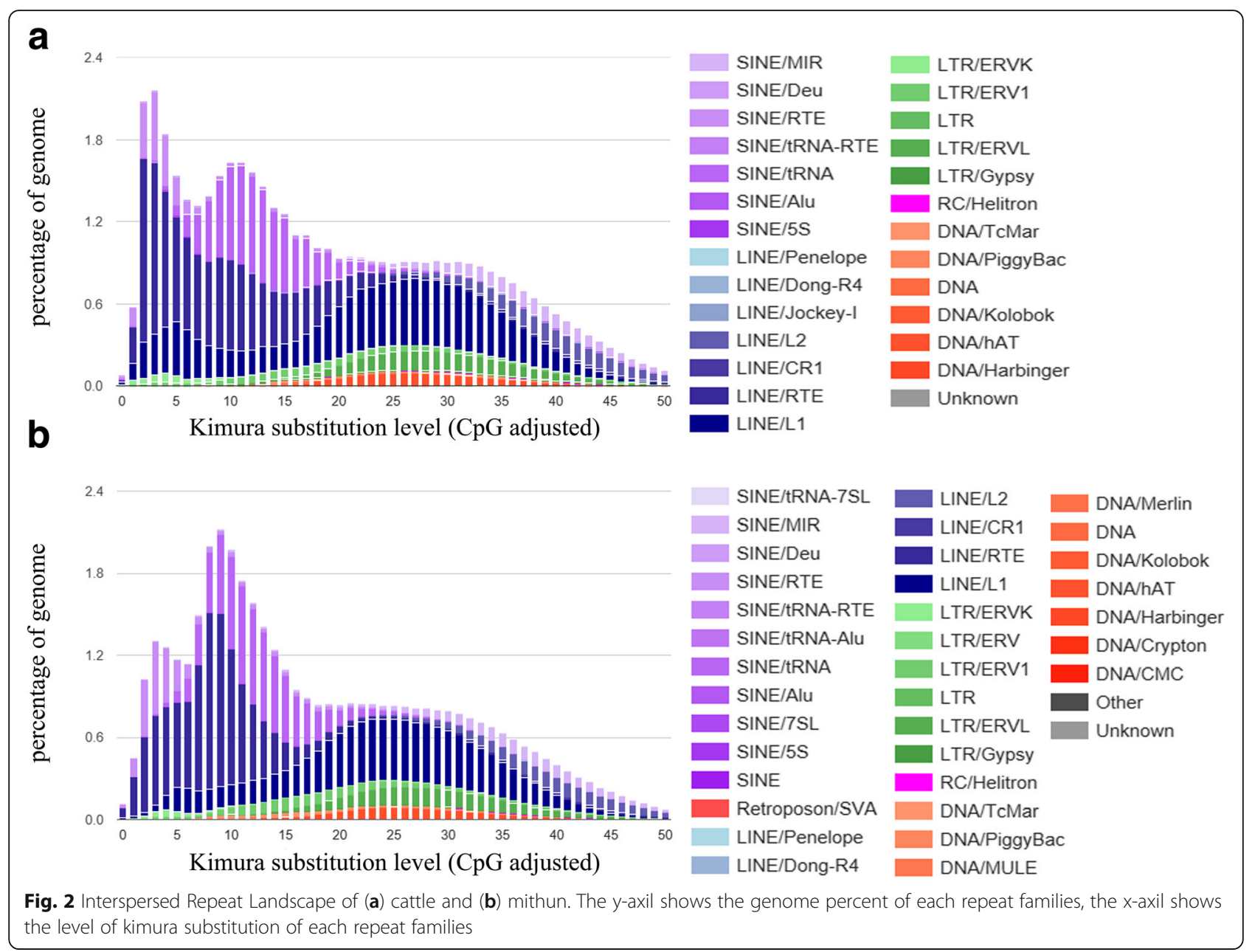

chicken [40], gray mouse lemur [39], gorilla [41] and Vibrio cholera [42] have previously been assembled using similar strategies, where PacBio long reads helped to assemble genome regions rich in repetitive elements. Follow up research showed that single molecular sequencing could reduce assembly complexity of microbial genome [43]. Even for large genomes like human, the hybrid strategy can markedly improve its contiguity [44]. We assembled the genome into 5,015 scaffolds, which is less fragmented and has more complete genome coverage than the previously published gayal assembly [8] (Table 1).

A number of processes viz. annotation of genome, combined homology search, $a b$ into prediction and a mithun transcriptome assembly [45] were used to identify protein-coding genes in the mithun genome. Genome annotation identified a high-quality set of 15,491 orthologous groups, a little less than cattle with about 16,000 groups. The number of gene orthologous families was lower than in other mammalian species (Fig. 2). Two primary reasons could account for this. Firstly, unlike chromosome-size scaffolds in cattle, the mithun genome assembly had smaller scaffolds. Because of this, some genes might be broken into two scaffolds and could not be detected. Secondly, we only had access to expression data from muscle tissue of mithun; therefore, we might have missed genes that were only expressed in other tissues or at different developmental stages. Nevertheless, our results greatly expand the information available on the gene sets present in the mithun genome.

\section{The origin and evolution of mithun}

Despite years of cytogenetic and phylogenetic studies, no consensus has been reached on the origin of mithun. Mithun was first classified as an independent species in 1968 [46]. This conclusion was recently supported by the cytochrome $b$ gene partial sequence [47]. Using mitochondrial DNA [48] and Y-chromosomal genes [46, 49], phylogenetic tree was constructed which showed a close relationship between the mithun and the gaur (Bos gaurus). This group was in turn found to be related to the banteng (Bos javanicus). Molecular phylogeny inferred from cytochrome $b$ (Cytb), subunit II of cytochrome c oxidase (CO2), and the promotor of the 


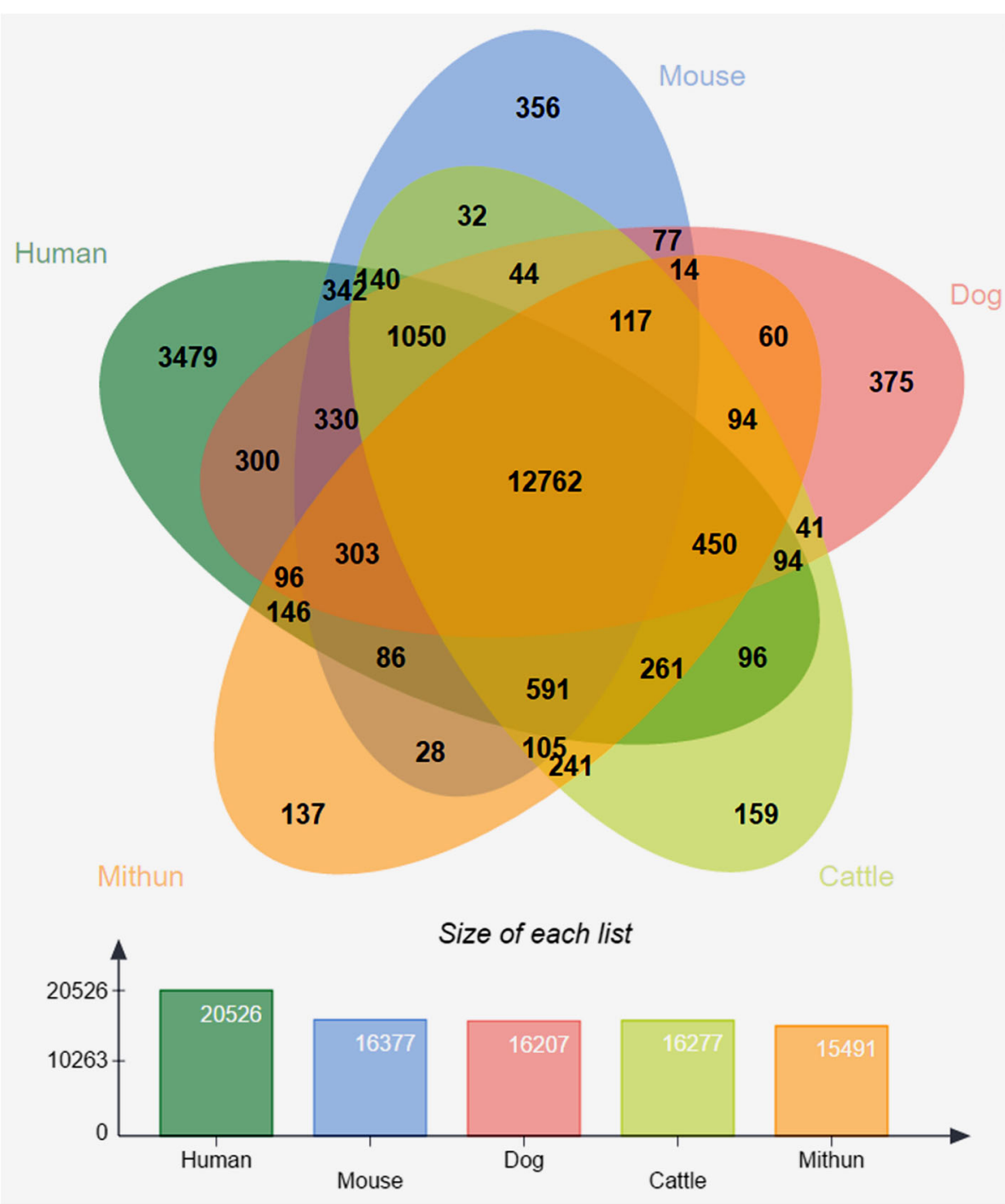

Fig. 3 The orthologous groups and evolution of mithun genes. The distinct and shared ortholog groups of mithun, mouse, dog, human and cattle detected by OrthoMCL

Table $\mathbf{5}$ The size distribution of synteny blocks of mithun genome aligned to cattle genome

\begin{tabular}{llllll}
\hline Block size & \multicolumn{2}{l}{ Mithun } & \multicolumn{2}{l}{ Gayal } \\
\cline { 2 - 3 } \cline { 5 - 6 } & Count of each block size & Total length for each block size $(\mathrm{bp})$ & & Count of each block size & Total length for each block size (bp) \\
\hline $10 \mathrm{bp}-100 \mathrm{bp}$ & 718 & 43,780 & 762 & 48,762 \\
$100 \mathrm{bp}-1 \mathrm{~kb}$ & 2,336 & 925,165 & 6,419 & $2,688,643$ \\
$1 \mathrm{~kb}-10 \mathrm{~kb}$ & 1,673 & $6,227,751$ & 3,940 & $11,741,636$ \\
$10 \mathrm{~kb}-100 \mathrm{~kb}$ & 3,918 & $204,420,988$ & 1,387 & $57,277,708$ \\
$100 \mathrm{~kb}-1 \mathrm{Mb}$ & 6,288 & $1,871,977,195$ & 2,373 & $1,007,062,584$ \\
$1 \mathrm{Mb}-10 \mathrm{Mb}$ & 156 & $201,022,481$ & 735 & $1,290,748,393$ \\
Total & & $2,588,165,483$ & $2,616,185,027$ & \\
\hline
\end{tabular}




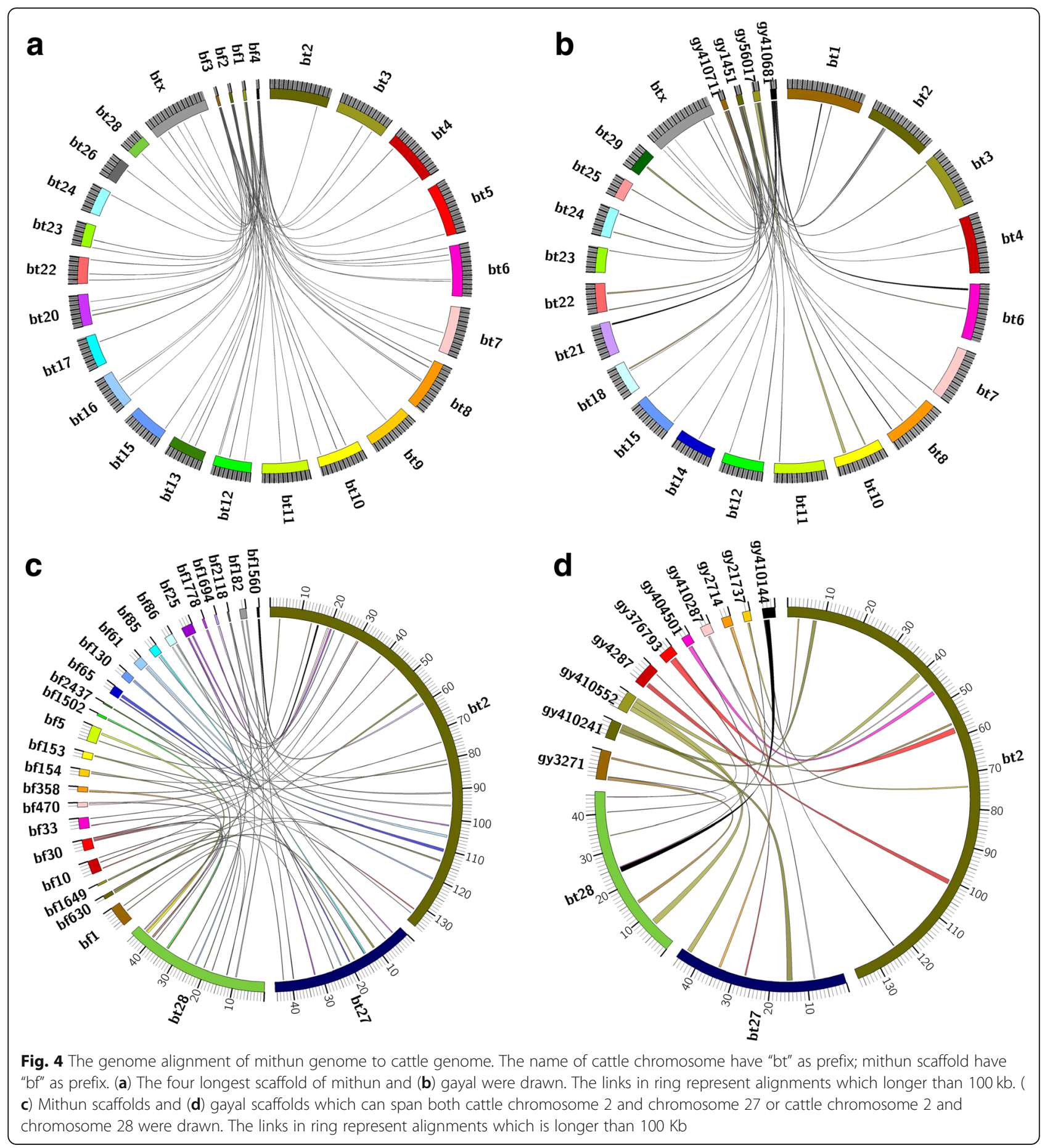

lactoferrin gene (Lf) clustered mithun and banteng into one clade [50]. The gaur was not included in that study. Based on cytogenetic evidence, mithun [51] and gaur $[50,52]$ were reported to share a Robertsonian translocation involving the homologs of cattle chromosomes 2 and 28 when compared to cattle. Another study suggested that compared with cattle, mithun have a speciesspecific $2 / 27$ centric fusion reducing the 60 chromosomes found in cattle to 58 chromosomes [6]. Neither of these conclusions was supported by our findings. To elucidate the origin of this unique species, either the chromosome level assembly of mithun (gayal) or sequencing mitochondrial genome of mithun taking a larger data set might be useful.

Patterns of genome-wide interspersed repeats in mithun showed both similarities to and differences from 
the pattern observed in cattle. The types of families observed in mithun and cattle were very similar. However, the distribution of the age of certain classes of repeat sequences clearly differed. Sequence divergence among LINEs and SINEs peaked at a much higher value in mithun than in cattle. This reflects that the numbers of LINEs and SINEs in mithun have expanded a relatively long time ago. In contrast, expansion of LINE and SINE numbers must have been much more recent in cattle. During the course of this study, we could see that the number of ancient repeat elements were very similar in these two species, but cattle have more repeat elements those are recently active (big peak of substitution level below 5 for cattle). Therefore, these two species have different evolution pattern after they diverged.

\section{Conclusion}

The main objective of the present study is to furnish a genetic resource and a de novo reference genome of mithun to facilitate future research. Our de novo draft assembly is the first genome assembly of Indian mithun, which is constructed using a hybrid approach. This improved the overall performance of the genome assembly. Our assembly is less fragmented, having better coverage and is completed to a reasonable extent. We believe this mithun genome assembly will provide genomic resource to evolutionary studies in combination with other bovine species, and will help to understand the genomic architecture of various phenotype and genotype interactions underlying this unique bovine species from distinct geographical habitat.

\section{Methods}

For sequencing of genomic DNA, blood sample was collected by a qualified Veterinarian in vacuutainer tube containing EDTA (Becton Dikinson, USA), from the jugular vein of one healthy adult female mithun $(2 \mathrm{n}=$ $58, \mathrm{XX})$, maintained in the Institute research farm, Medziphema, Nagaland, India under semi-intensive rearing system. The standard animal ethics normswere followed and care of the animal was taken in accordance with guidelines of the Committee for the Purpose of Control and Supervision on Experiments on Animals (CPCSEA), prescribed by the Indian Council of Agricultural Research (ICAR), Ministry of Agriculture and Farmers Welfare, Government of India. In an earlier study on gene expression, the muscle samples were collected for RNA extraction from growing male mithuns, average 24 months of age (range 19-29 months) under standard anaesthesia by a qualified Veterinary Surgeon, from the Institute Research Farm, Medziphema. Institutional Animal Ethics Committee had approved collection of mithun blood and muscle samples for the purpose of DNA and RNA extraction. All these procedures under the present study agrees with the ARRIVE Guidelines for reporting research [53] involving animals (Additional file 1).

\section{DNA isolation, libraries preparation and sequencing Paired-end sequencing}

Genomic DNA from blood was prepared using QIAamp DNA Mini Kit (Qiagen) and was quantified using Qubit DNA BR Quantitation kit (Invitrogen). The genomic library was prepared according to the manufacturer's protocol (Illumina, True Seq DNA preparation guide) using the Illumina TruSeq DNA LT library kit. The paired-end library was sequenced on an Illumina HiSeq 2500 in $2 \times 100$ cycles using the SBS sequencing kits V3.0, generating a total of $201.06 \mathrm{~Gb}$ of paired-end data. These sequence data was submitted in NCBI Database (BioProject ID PRJNA241403).

\section{Mate-pair sequencing}

Following fragmentation, the DNA fragments were endrepaired with labeled dNTPs. The DNA fragments were circularized, and non-circularized DNA was removed by digestion. Circular DNA was fragmented, and the labeled fragments (corresponding to the ends of the original DNA ligated together) were purified using affinity chromatography. Purified fragments were end-repaired and ligated to Illumina paired-end sequencing adapters. Additional sequences complementary to the flow cell oligonucleotides were added to the adapter sequence with tailed PCR primers. The final libraries prepared in this process were consisted of short fragments made up of two DNA segments, originally separated by several kilobases. Two separate mate-pair libraries of $3 \mathrm{~kb}$ each and three libraries of $5 \mathrm{~kb}$ each were prepared using Illumina Nextera Mate-Pairs sample preparation kit as per manufacturer's protocol. These libraries were then sequenced using Illumina NGS platform (HiSeq 2500) to generate a total of $40.40 \mathrm{~Gb}$ high quality and cleaned mate-pair sequence reads.

\section{Illumina moleculo long-reads}

Illumina TruSeq synthetic long-read technology was used to generate moleculo long reads in this study. The protocol involves initial mechanical fragmentation of genomic DNA into $10 \mathrm{~kb}$ fragments. These fragments then undergo end-repair and ligation of amplification adapters, before diluted onto 384-well plates so that each well contains DNA representing approximately $1-2 \%$ of the genome (200 molecules, in the case of D. melanogaster). Polymerase chain reaction (PCR) was used to amplify molecules within wells, followed by parallel Nexterabased fragmentation and barcoding of individual wells. DNA from all wells was then pooled and sequenced on the Illumina HiSeq 2000 platform. Data from individual 
wells were demultiplexedin silico according by barcode sequences, generating approx. 4.4 Gb clean sequence data. Synthetic long-reads were assembled from the short reads using a specific assembly pipeline.

\section{PacBio sequencing}

PacBio (Pacific BioSciences) long read sequencing technique, enabled by the SMRTbell ${ }^{\circ}$ (Single-molecule Real Time) technology was employed in this study. The SMRTbell ${ }^{\circ}$ template preparation method creates a circularized template for use with multiple sequencing protocols. A single streamlined protocol was used to create different insert size libraries i.e. $10 \mathrm{~kb}$ and $20 \mathrm{~kb}$ by altering the fragmentation conditions. The first step in the generation of a SMRTbell library was production of appropriately sized double-stranded DNA fragments. These fragments can be generated by random shearing of DNA, or by amplification of target regions of interest. The SMRTbell library was produced by ligating universal hairpin adapters onto double-stranded DNA fragments. The hairpin dimers formed during this process were removed at the end of the protocol using a magnetic bead purification step with size-selective conditions. The final step of the protocol was to remove failed ligation products with exonucleases. After the exonuclease step, SMRTbell templates were annealed to primers, and annealed templates were bound to DNA polymerase. Lastly, the sample plate was set up for sequencing.

\section{RNA extraction, CDNA synthesis, library preparation and sequencing}

RNA was extracted from each of the four muscle tissues following standard guidelines of Illumina Low Sample Protocol (TruSeq ${ }^{\bullet}$ RNA Sample Preparation v2 Guide). In brief, total RNA integrity following isolation was checked using an Agilent Technologies 2100 Bioanalyzer for each sample with an RNA Integrity Number (RIN) value greater than or equal to eight. The first step in the workflow involved purifying the poly-A containing mRNA molecules using poly-T oligo-attached magnetic beads. Following purification, the mRNA was fragmented into small pieces using divalent cations under elevated temperature. The cleaved RNA fragments were copied into first strand cDNA using reverse transcriptase and random primers. This was followed by second strand cDNA synthesis using DNA Polymerase I and RNase $H$. These cDNA fragments then went through an end repair process, the addition of a single ' $\mathrm{A}$ ' base, and then ligation of the adapters. The products were then purified and enriched with PCR to create the final cDNA library. This protocol for transcriptome analysis was performed on RNA after mRNA purification using elevated temperatures, resulting in libraries with insert size ranging from 120 to $200 \mathrm{bp}$ with a median size of $150 \mathrm{bp}$.
Transcriptome sequencing was carried out using the Illumina Hi-seq 2000 platform to generate paired-end reads. The RNAseq data are deposited in the NCBI Database (BioProject accessions: PRJNA307305; BioSample accessions: SAMN04384021, SAMN04384020, SAMN04384019 and SAMN04384018).

\section{Genomic data processing, genome assemble and assembly assessment}

Trimmomatic [54] was used to remove the adaptor and trim the raw data of Illumina paired-end (PE) sequencing and mate-pair (MP) sequencing data. High quality PE data were assembled by MaSuRCA [53]. The contigs obtained from MaSuRCA and cleaned MP data were scaffold by SSPACE [17]. PacBio data were error-corrected and trimmed by LoRDEC [55] by using error corrected PE data. Then we re-scaffolded the assembly by SSPACE-Long Read with error corrected PacBio data and raw illumine Moleculo Long reads. Thereafter, the assembly was polished by PBJelly 2 [56]. Final statistics of the assembly were assessed by QUAST [57]. Part of the PE and MP data were mapped to the draft genome by BWA [58]. Properly paired reads reported by samtools [59] flagstat were used to investigate the correctness of assembly. BUSCO v3 $[20,60]$ with the mammalian database was used to assess the completeness of genes presented by assembly. Nineteen-mers was counted from PE data with Jellyfish [61]. Genome size was estimated by dividing the total number of $k$-mers by the peak value of the $\mathrm{k}$-mer frequency distribution [62].

\section{RNA-seq data processing}

RNA-seq data having adaptor sequences were removed and low-quality bases (average quality per base drops below 15 in 4 bases sliding windows) were trimmed using Trimmomatic [54]. RNA-seq data from four samples were mixed together to help to build a comprehensive muscle transcriptome. The mixed data set were de novo assembled by Trinity [63]. We also performed genome guided assembly by following procedure: the mixed dataset were aligned to genome assembly by Tophat2 [64]. Trinity then assembled the aligned reads. We also generated the transcriptome by Cufflink [65].

\section{Repeat sequence annotation}

The genome assembly were masked by RepeatMasker [23] with the mammalian database. The substitution level (alignment of each repeat element sequences with their consensus sequence in database) calculation and plots were done using calcDivergenceFromAlign.pl and createRepeatLandscape.pl scripts provided with RepeatMasker. The RepeatLandscape for cattle genome assembly (BTA7) was downloaded from the RepeatMasker website of preanalysis species. The statistics for the BTA7 assembly was 
also downloaded from RepeatMasker website and compared with mithun.

\section{Gene annotation}

We annotated the mithun genome by combination of three strategies: Ab Initio gene prediction, protein homology search and a transcriptome assembly. Homology search was scanned by Exonerate [66] against mammalian protein sequences collected from Uniport [67]. Trinity [63] de novo assembly, Trinity [63] genome-guided assembly and TopHat2 plus Cufflinks [64] assembly was merged to build a comprehensive transcriptome by PASA pipeline [68]. Ab initio gene prediction was performed by Augustus [69], using the configure file trained by BUSCO [20]. In addition, we provided the RepeatMasker [23], Exonerate [66], PASApipeline [68] and Tophat2 [64] alignment of RNA-seq data as hints for Augustus [69]. These three set of annotations were merged by EVM [70] by weighting them as ab initio gene prediction: 1 ; Homology search: 6 and transcriptome: 10 as suggested in the EVM manual [70]. For mithun reference genes, motifs and domains were detected by InterProScan [26] against multiple database including Pfam [71], Panther [72], PRINTS [73], Gene3D [74], SUPERFAMILY [75]. The GO terms of each gene was assigned by eggNOGmapper [25]. We also used KASS [76] to identify the KEGG [77] pathway information of [77] pathway information of the mithun gene set.

\section{Genome alignment}

Both mithun and gayal genomes were soft-masked and aligned to the soft-masked cattle genome (ARS-UCD1.2) [21] by Large Scale Genome Alignment Tools (LASTZ) [78]. The pairwise genome alignment was chained according to their location in both genomes by axtChain program [79]. The netting process chooses for the reference species the best sub-chain in each region. The statistics of different size of synteny block was done by a custom script. We only used block size larger than 100 $\mathrm{kb}$ to investigate how many cattle chromosomes the mithun scaffold can span.

\section{Additional files}

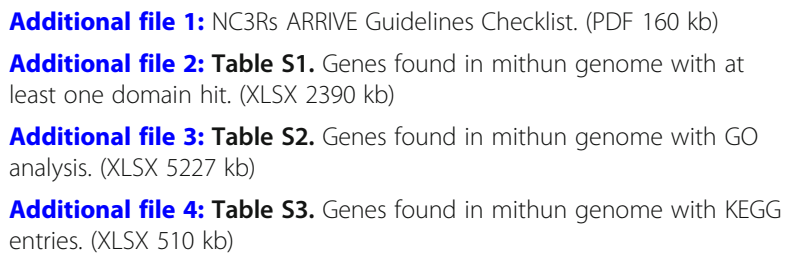

\section{Abbreviations}

BTA 7: Bos taurus genome assembly v.7; BUSCO: Benchmarking Universal Single-Copy Orthologs; CNVs: Copy number variations; DBG: De Bruijn
Graphs; dNTPs: Deoxyribonucleotide triphosphates; eggNOG: Evolutionary genealogy of genes: Non-supervised Orthologous Groups; EVM: EVidence Modeler; Gb: Gigabyte; KAAS: KEGG Automatic Annotation Server; KEGG: Kyoto Encyclopedia of Genes and Genomes; LASTZ: Large-Scale Genome Alignment Tool; LINEs: Long Interspersed Nuclear Elements; LORDEC: Long Read DBG Error Correction; LTR: Long terminal repeat; MaSuRCA: Maryland Super-Read Celera Assembler; MB: Megabyte; Mt:: Mitochondria; NGS: Next-generation sequencing; PacBio: Pacific BioSciences; PASApipeline: Program to Assemble Spliced Alignments Pipeline; PBJelly 2: Software for Long-Read Sequencing Data from PacBio; Pfam: Protein families database; PRINTS: Protein fingerprints database; QUAST: Quality Assessment Tools; RIN: RNA Integrity Number; SINEs: Short Interspersed Nuclear Elements; SMRT: Single-molecule real-time (SMRT); SNPs: Single-Nucleotide Polymorphisms; SSPACE: Scaffolding Pre-Assemblies AfterContig Extension; SVs: Structural variations

\section{Acknowledgements}

The authors would like to thank the Director of the Institute and Indian Council of Agricultural Research for funding the Institutional Research Project for this study. Financial assistance in the form of DBT Overseas Associateship for the first author is gratefully acknowledged. IL and MM were supported by a DST and DBT funded grant, Govt. Of India, respectively. Assistance of DBT Biotech Hub in carrying out a part of wet lab work is thankfully acknowledged.

\section{Authors' contributions}

SM, AM and CR conceived the study. SM, ZC and AM run analysis and wrote the manuscript consulting other authors. IL, MM, KV, KK collected samples and run wet lab work. $\mathrm{ABM}, \mathrm{BG}, \mathrm{MS}$, and GS critically read and revised the manuscript. All authors read and approved the final manuscript.

\section{Funding}

This work was carried out under an Institutional research project funded by ICAR-NRC on Mithun, Nagaland, India. Financial support in the form of DBT Overseas Associateship for the first author played a significant role to carry out some of the bioinformatic analyses and their interpretations at Aarhus University, Denmark.

\section{Availability of data and materials}

All data generated and analyzed during this current study are available in the Data Cell of ICAR-NRC on Mithun, Nagaland, India with permission from the Competent Authority. Illumina whole genome sequence and RNAseqdata of mithun were submitted in NCBI Database having BioProject ID PRJNA241403 and BioProject ID PRJNA307305, respectively.

Ethics approval and consent to participate

All the animal experiments and sample collections procedures were carried out by qualified Veterinarians of the Institute. The ethics approval for animal experimentation was obtained from the Institute Ethics Approval Committee under the mandatory regulations of CPCSEA (Committee for the Purpose of Control and Supervision on Experiments on Animals) guidelines, prescribed by the Indian Council of Agricultural Research (ICAR), Ministry of Agriculture and Farmers Welfare, Government of India. Since no specimen was used, no permissions were necessary to collect the specimens in our present study.

Consent for publication

Not Applicable

\section{Competing interests}

The authors declare that they have no competing interests.

\section{Author details}

${ }^{1}$ Animal Genetics and Breeding Lab., ICAR-National Research Centre on Mithun, Medziphema, Nagaland 797106, India. ${ }^{2}$ Center for Quantitative Genetics and Genomics, Department of Molecular Biology and Genetics, Aarhus University, 8830 Tjele, Denmark. ${ }^{3}$ Present address: Dairy Cattle Breeding Division, ICAR-National Dairy Research Institute, Karnal, Haryana 132001, India. 


\section{Received: 18 June 2018 Accepted: 16 July 2019}

\section{Published online: 29 July 2019}

\section{References}

1. Mondal M, Dhali A, Rajkhowa C, Prakash BS. Secretion patterns of growth hormone in growing captive mithuns (Bos frontalis). Zool Sci. 2004:21(11):1125-9.

2. Gupta N, Verma ND, Gupta SC, Kumar P, Sahai R. Chromosomes of Mithun (Gaveaus-frontalis). Indian J An Sci. 1995;65(6):688-90.

3. FJaS S, Elizabeth S. a ceremonial ox of India: the mithun in nature, culture, and history, with notes on the domestication of common cattle. Madison, Wl: The University of Wisconsin Press; 1968.

4. Tanaka K, Takizawa T, Murakoshi H, Dorji T, Nyunt MM, Maeda Y, Yamamoto Y, Namikawa T. Molecular phylogeny and diversity of Myanmar and Bhutan mithun based on mtDNA sequences. Anim Sci J. 2011:82(1):52-6.

5. Lan H, Xiong X, Lin S, Liu A, Shi L. Mitochondrial DNA polymorphism of cattle (Bos taurus) and mithun (Bos frontalis) in Yunnan Province. Yi chuan xue bao=. Acta Genet Sin. 1992;20(5):419-25.

6. Winter H, Mayr B, Schleger W, Dworak E, Krutzler J, Kalat M. Genetic characterisation of the mithun (Bos frontalis) and studies of spermatogenesis, blood groups and haemoglobins of its hybrids with Bos indicus. Res Vet Sci. 1986:40(1):8-17.

7. Mei C, Wang H, Zhu W, Wang H, Cheng G, Qu K, Guang X, Li A, Zhao C, Yang $W$, et al. Whole-genome sequencing of the endangered bovine species Gayal (Bos frontalis) provides new insights into its genetic features Sci Rep. 2016:6:19787.

8. Wang MS, Zeng Y, Wang X, Nie WH, Wang JH, Su WT, Otecko NO, Xiong ZJ, Wang S, Qu KX, et al. Draft genome of the gayal. Bos frontalis Gigascience. 2017;6(11):1-7.

9. Bovine Genome S, Analysis C, Elsik CG, Tellam RL, Worley KC, Gibbs RA, Muzny DM, Weinstock GM, Adelson DL, Eichler EE, et al. The genome sequence of taurine cattle: a window to ruminant biology and evolution. Science. 2009:324(5926):522-8.

10. Weldenegodguad M, Popov R, Pokharel K, Ammosov I, Ming Y, Ivanova Z and Kantanen J. 2019. Whole-Genome Sequencing of Three Native Cattle Breeds Originating From the Northernmost Cattle Farming Regions. Front. Genet. Published online 2019. https://doi.org/10.3389/fgene.2018.00728.

11. Tantia MS, Vijh RK, Bhasin V, Sikka P, Vij PK, Kataria RS, Mishra BP, Yadav SP, Pandey AK, Sethi RK, et al. Whole-genome sequence assembly of the water buffalo (Bubalus bubalis). Indian J Animal Sci. 2011;81(5):465-73.

12. Qiu Q, Zhang G, Ma T, Qian W, Wang J, Ye Z, Cao C, Hu Q, Kim J, Larkin DM, et al. The yak genome and adaptation to life at high altitude. Nat Genet. 2012;44(8):946-9.

13. McCoy RC, Taylor RW, Blauwkamp TA, Kelley JL, Kertesz M, Pushkarev D, Petrov DA, Fiston-Lavier AS. Illumina TruSeq synthetic long-reads empower de novo assembly and resolve complex, highly-repetitive transposable elements. PLoS One. 2014;9(9):e106689.

14. Caporaso JG, Lauber CL, Walters WA, Berg-Lyons D, Huntley J, Fierer N, Owens SM, Betley J, Fraser L, Bauer M, et al. Ultra-high-throughput microbial community analysis on the Illumina HiSeq and MiSeq platforms. ISME J. 2012;6(8):1621-4

15. Rhoads A, Au KF. PacBio sequencing and its applications. Genomics Proteomics Bioinformatics. 2015;13(5):278-89.

16. Zimin AV, Marcais G, Puiu D, Roberts M, Salzberg SL, Yorke JA. The MaSuRCA genome assembler. Bioinformatics. 2013;29(21):2669-77.

17. Boetzer M, Henkel CV, Jansen HJ, Butler D, Pirovano W. Scaffolding preassembled contigs using SSPACE. Bioinformatics. 2011;27(4):578-9.

18. Salmela L, Rivals E. LoRDEC: accurate and efficient long read error correction. Bioinformatics. 2014;30(24):3506-14.

19. Boetzer M, Pirovano W. SSPACE-LongRead: scaffolding bacterial draft genomes using long read sequence information. BMC Bioinformatics. 2014;15(1):211.

20. Simao FA, Waterhouse RM, loannidis P, Kriventseva EV, Zdobnov EM BUSCO: assessing genome assembly and annotation completeness with single-copy orthologs. Bioinformatics. 2015;31(19):3210-2.

21. Zimin AV, Delcher AL, Florea L, Kelley DR, Schatz MC, Puiu D, Hanrahan F, Pertea G, Van Tassell CP, Sonstegard TS, et al. A whole-genome assembly of the domestic cow, Bos taurus. Genome Biol. 2009:10(4):R42

22. Canavez FC, Luche DD, Stothard P, Leite KR, Sousa-Canavez JM, Plastow G, Meidanis J, Souza MA, Feijao P, Moore SS, et al. Genome sequence and assembly of Bos indicus. J Hered. 2012;103(3):342-8.
23. Tarailo-Graovac M, Chen N: Using RepeatMasker to identify repetitive elements in genomic sequences. Curr Protoc Bioinformatics 2009:4.10. 11-14.10. 14.

24. Bao W, Kojima KK, Kohany O. Repbase update, a database of repetitive elements in eukaryotic genomes. Mob DNA. 2015;6(1):11.

25. Huerta-Cepas J, Forslund K, Coelho LP, Szklarczyk D, Jensen LJ, von Mering C, Bork P. Fast Genome-wide functional annotation through Orthology assignment by eggNOG-mapper. Mol Biol Evol. 2017;34(8):2115-22.

26. Zdobnov EM, Apweiler R. InterProScan-an integration platform for the signature-recognition methods in InterPro. Bioinformatics. 2001;17(9):847-8.

27. Ashburner M, Ball CA, Blake JA, Botstein D, Butler H, Cherry JM, Davis AP, Dolinski K, Dwight SS, Eppig JT, et al. Gene ontology: tool for the unification of biology. The gene ontology Consortium. Nat Genet. 2000;25(1):25-9.

28. Kanehisa M, Goto S. KEGG: Kyoto encyclopedia of genes and genomes. Nucleic Acids Res. 2000:28(1):27-30

29. Moriya Y, Itoh M, Okuda S, Yoshizawa AC, Kanehisa M: KAAS: an automatic genome annotation and pathway reconstruction server. Nucleic Acids Res 2007, 35(suppl_2):W182-W185.

30. Prakash B, Dhali A, Rathore SS, Das KC, Walling I, Vupru K, Mech A, Baruah KK, Rajkhowa C. Chemical composition and nutritional evaluation of various foliages consumed by mithun (Bos frontalis). Anim Feed Sci Technol. 2009;150(3-4):223-9.

31. Mondal M, Baruah K, Rajkhowa C. Mithun: an animal of Indian pride. Lives Res Rural Deve. 2014;26.

32. Xi D, Liu Q, Guo J, Yu H, Yang Y, He Y, Mao H, Gou X, Deng W. Genetic variability of the coding region for the prion protein gene (PRNP) in gayal (Bos frontalis). Mol Biol Rep. 2012;39(2):2011-20.

33. Ross MG, Russ C, Costello M, Hollinger A, Lennon NJ, Hegarty R, Nusbaum $C$, Jaffe DB. Characterizing and measuring bias in sequence data. Genome Biol. 2013:14(5):R51.

34. Ikegami T, Inatsugi T, Kojima I, Umemura M, Hagiwara H, Machida M, Asai K Hybrid De Novo Genome Assembly Using MiSeq and SOLiD Short Read Data. PLoS One. 2015;10(4):e0126289.

35. Miller JR, Zhou P, Mudge J, Gurtowski J, Lee H, Ramaraj T, Walenz BP, Liu J, Stupar RM, Denny R, et al. Hybrid assembly with long and short reads improves discovery of gene family expansions. BMC Genomics. 2017;18(1):541

36. English AC, Richards S, Han Y, Wang M, Vee V, Qu J, Qin X, Muzny DM, Reid JG, Worley KC, et al. Mind the gap: upgrading genomes with Pacific Biosciences RS long-read sequencing technology. PLoS One. 2012;7(11):e47768.

37. Mostovoy Y, Levy-Sakin M, Lam J, Lam ET, Hastie AR, Marks P, Lee J, Chu C, Lin C, Dzakula Z, et al. A hybrid approach for de novo human genome sequence assembly and phasing. Nat Methods. 2016;13(7):587-90.

38. Putnam NH, O'Connell BL, Stites JC, Rice BJ, Blanchette M, Calef R, Troll CJ, Fields A, Hartley PD, Sugnet CW, et al. Chromosome-scale shotgun assembly using an in vitro method for long-range linkage. Genome Res. 2016:26(3):342-50.

39. Larsen PA, Harris RA, Liu Y, Murali SC, Campbell CR, Brown AD, Sullivan BA, Shelton J, Brown SJ, Raveendran M, et al. Hybrid de novo genome assembly and centromere characterization of the gray mouse lemur (Microcebus murinus). BMC Biol. 2017:15(1):110.

40. Sohn J-i, Nam K, Kim J-M, Lim D, Lee K-T, Do YJ, Cho CY, Kim N, Nam J-W, Chae $\mathrm{H}-\mathrm{H}$ : Whole genome hybrid assembly and protein-coding gene annotation of the entirely black native Korean chicken breed Yeonsan Ogye bioRxiv 2017:224311.

41. Gordon D, Huddleston J, Chaisson MJ, Hill CM, Kronenberg ZN, Munson KM, Malig M, Raja A, Fiddes I, Hillier LW, et al. Long-read sequence assembly of the gorilla genome. Sci. 2016;352(6281):aae0344

42. Bashir A, Klammer AA, Robins WP, Chin C-S, Webster D, Paxinos E, Hsu D, Ashby M, Wang S, Peluso P. A hybrid approach for the automated finishing of bacterial genomes. Nat Biotechnol. 2012;30(7):701

43. Koren S, Harhay GP, Smith TP, Bono JL, Harhay DM, McVey SD, Radune D, Bergman NH, Phillippy AM. Reducing assembly complexity of microbial genomes with single-molecule sequencing. Genome Biol. 2013;14(9):R101.

44. Pendleton M, Sebra R, Pang AW, Ummat A, Franzen O, Rausch T, Stutz AM, Stedman W, Anantharaman T, Hastie A, et al. Assembly and diploid architecture of an individual human genome via single-molecule technologies. Nat Methods. 2015;12(8):780-6.

45. Mukherjee S, Mukherjee A, Jasrotia RS, Jaiswal S, lquebal MA, Longkumer I, Mech M, Vüpru K, Khate K, Rajkhowa C, et al. Muscle transcriptome 
signature and gene regulatory network analysis in two divergent lines of a hilly bovine species Mithun (Bos frontalis). Genomics. 2019.

46. Walker EP: Mammals of the world, vol. III. Hopkins, Baltimore 1968

47. Ma G, Chang H, Li S, Chen H, Ji D, Geng R, Chang C, Li Y. Phylogenetic relationships and status quo of colonies for gayal based on analysis of cytochrome B gene partial sequences. J Genet Genomics. 2007;34(5):413-9.

48. Verkaar EL, Nijman IJ, Beeke M, Hanekamp E, Lenstra JA. maternal and paternal lineages in cross-breeding bovine species. Has wisent a hybrid origin. Mol Biol Evol. 2004;21(7):1165-70.

49. Nijman IJ, van Boxtel DCJ, van Cann LM, Marnoch Y, Cuppen E, Lenstra JA Phylogeny of $Y$ chromosomes from bovine species. Cladistics. 2008;24(5):723-6.

50. Hassanin A, Ropiquet A. Molecular phylogeny of the tribe Bovini (Bovidae, Bovinae) and the taxonomic status of the Kouprey, Bos sauveli Urbain 1937. Mol Phylogenet Evol. 2004;33(3):896-907.

51. Chi J, Fu B, Nie W, Wang J, Graphodatsky AS, Yang F. New insights into the karyotypic relationships of Chinese muntjac (Muntiacus reevesi), forest musk deer (Moschus berezovskii) and gayal (Bos frontalis). Cytogenet Genome Res. 2005:108(4):310-6.

52. Gallagher D Jr, Womack J. Chromosome conservation in the Bovidae. J Hered. 1992;83(4):287-98.

53. Kilkenny C, Browne WJ, Cuthill IC, Emerson M, Altman DG. Improving bioscience research reporting: the ARRIVE guidelines for reporting animal research. PLoS Biol. 2010;8:e1000412.

54. Bolger AM, Lohse M, Usadel B. Trimmomatic: a flexible trimmer for Illumina sequence data. Bioinformatics. 2014;30(15):2114-20.

55. LoRDEC: a hybrid error correction program for long, PacBio reads. Assessed online. http://atgc.lirmm.fr/lordec/.

56. Worley KC: Improving Genomes Using Long Reads and PBJelly 2. In: Plant and Animal Genome XXII Conference: 2014: Plant and Animal Genome; 2014.

57. Gurevich A, Saveliev V, Vyahhi N, Tesler G. QUAST: quality assessment tool for genome assemblies. Bioinformatics. 2013;29(8):1072-5.

58. Li H: Aligning sequence reads, clone sequences and assembly contigs with BWA-MEM. arXiv preprint arXiv:13033997 2013.

59. Li H. A statistical framework for SNP calling, mutation discovery, association mapping and population genetical parameter estimation from sequencing data. Bioinformatics. 2011;27(21):2987-93.

60. Waterhouse RM, Seppey M, Simão FA, Manni M, loannidis P, Klioutchnikov G, Kriventseva EV, and Zdobnov EM. BUSCO applications from quality assessments to gene prediction and phylogenomics. Mol Biol Evol, published online Dec 6, 2017. https://doi.org/10.1093/molbev/ms×319.

61. Marcais $G$, Kingsford C. A fast, lock-free approach for efficient parallel counting of occurrences of k-mers. Bioinformatics. 2011;27(6):764-70.

62. Guo LT, Wang SL, Wu QJ, Zhou XG, Xie W, Zhang YJ. Flow cytometry and K-mer analysis estimates of the genome sizes of Bemisia tabaci B and $Q$ (Hemiptera: Aleyrodidae). Front Physiol. 2015;6:144.

63. Haas BJ, Papanicolaou A, Yassour M, Grabherr M, Blood PD, Bowden J, Couger MB, Eccles D, Li B, Lieber M, et al. De novo transcript sequence reconstruction from RNA-seq using the trinity platform for reference generation and analysis. Nat Protoc. 2013:8(8):1494-512.

64. Kim D, Pertea G, Trapnell C, Pimentel H, Kelley R, Salzberg SL. TopHat2: accurate alignment of transcriptomes in the presence of insertions, deletions and gene fusions. Genome Biol. 2013;14(4):R36.

65. Trapnell C, Roberts A, Goff L, Pertea G, Kim D, Kelley DR, Pimentel H, Salzberg SL, Rinn JL, Pachter L. Differential gene and transcript expression analysis of RNA-seq experiments with TopHat and cufflinks. Nat Protoc. 2012;7(3):562-78.

66. Slater GS, Birney E. Automated generation of heuristics for biological sequence comparison. BMC Bioinformatics. 2005;6(1):31.

67. Consortium U. The universal protein resource (UniProt). Nucleic Acids Res. 2008;36(suppl 1):D190-5.

68. Haas BJ, Delcher AL, Mount SM, Wortman JR, Smith RK, Hannick LI, Maiti R, Ronning CM, Rusch DB, Town CD, et al. Improving the Arabidopsis genome annotation using maximal transcript alignment assemblies. Nucleic Acids Res. 2003;31(19):5654-66.

69. Stanke M, Keller O, Gunduz I, Hayes A, Waack S, Morgenstern B. AUGUSTUS: ab initio prediction of alternative transcripts. Nucleic Acids Res. 2006;34(Web Server issue):W435-9.

70. Haas BJ, Salzberg SL, Zhu W, Pertea M, Allen JE, Orvis J, White O, Buell CR, Wortman JR. Automated eukaryotic gene structure annotation using
EVidenceModeler and the program to assemble spliced alignments. Genome Biol. 2008:9(1):R7.

71. Bateman A, Coin L, Durbin R, Finn RD, Hollich V, Griffiths-Jones S, Khanna A, Marshall M, Moxon S, Sonnhammer EL, et al. The Pfam protein families database. Nucleic Acids Res. 2004;32(Database issue):D138-41.

72. Thomas PD, Campbell MJ, Kejariwal A, Mi H, Karlak B, Daverman R, Diemer K, Muruganujan A, Narechania A. PANTHER: a library of protein families and subfamilies indexed by function. Genome Res. 2003;13(9):2129-41.

73. Attwood TK, Blythe MJ, Flower DR, Gaulton A, Mabey JE, Maudling N, McGregor L, Mitchell AL, Moulton G, Paine K, et al. PRINTS and PRINTS-S shed light on protein ancestry. Nucleic Acids Res. 2002;30(1):239-41.

74. Yeats C, Maibaum M, Marsden R, Dibley M, Lee D, Addou S, Orengo CA. Gene3D: modelling protein structure, function and evolution. Nucleic Acids Res. 2006;34(Database issue):D281-4.

75. Wilson D, Pethica R, Zhou Y, Talbot C, Vogel C, Madera M. SUPERFAMILYcomparative genomics, datamining and sophisticated visualization. Nucleic Acids Res. 2009:37:D380-6.

76. Moriya Y, Itoh M, Okuda S, Yoshizawa AC, Kanehisa M. KAAS: an automatic genome annotation and pathway reconstruction server. Nucleic Acids Res. 2007:35(Web Server issue):W182-5.

77. Okuda S, Yamada T, Hamajima M, Itoh M, Katayama T, Bork P, Goto S, Kanehisa M. KEGG Atlas mapping for global analysis of metabolic pathways. Nucleic Acids Res. 2008;36(Web Server issue):W423-6.

78. Harris RS. Improved pairwise alignment of genomic DNA: the Pennsylvania State University; 2007.

79. Kent WJ, Baertsch R, Hinrichs A, Miller W, Haussler D. Evolution's cauldron: duplication, deletion, and rearrangement in the mouse and human genomes. Proc Natl Acad Sci U S A. 2003;100(20):11484-9.

\section{Publisher's Note}

Springer Nature remains neutral with regard to jurisdictional claims in published maps and institutional affiliations.

Ready to submit your research? Choose BMC and benefit from:

- fast, convenient online submission

- thorough peer review by experienced researchers in your field

- rapid publication on acceptance

- support for research data, including large and complex data types

- gold Open Access which fosters wider collaboration and increased citations

- maximum visibility for your research: over $100 \mathrm{M}$ website views per year

At $\mathrm{BMC}$, research is always in progress.

Learn more biomedcentral.com/submissions 